\section{Prevalence of depressive symptoms amongst highly active antiretroviral therapy (HAART) patients in AIDSRelief Uganda}

\author{
Constance Shumba, ${ }^{1}$ Ruth Atukunda, ${ }^{1}$ \\ Richard Imakit, ${ }^{1}$ Peter Memiah ${ }^{2}$ \\ ${ }^{1}$ Uganda program, Institute of Human \\ Virology, University of Maryland, \\ Kampala Uganda; ${ }^{2}$ Institute of Human \\ Virology, University of Maryland, \\ Baltimore, MD, USA
}

\section{Abstract}

There is limited data on the prevalence of depression in HIV and AIDS patients in SubSaharan Africa and little resources have been allocated to address this issue. Depression affects patient adherence to treatment and predisposes patients to resistance which poses a public health threat. It also affects quality of life and productivity of patients. From August 2008 to March 2009, 731 patient adherence surveys were administered to assess disease, treatment knowledge and services received. The primary variable of interest was patients' level of depressive symptoms score, constructed using factor analysis from five survey questions relating to: sadness, need to be alone, hopelessness and confusion and was categorized as no depressive symptoms (score 0), low depressive symptoms (score 1-2), moderate depressive symptoms (score 3-4) and high depressive symptoms (score 5-10). Majority of the patients on highly active antiretroviral therapy (HAART) (59\%) were found to have depressive symptoms and this was more among women than men $(66 \%$ vs $43 \%)$. There was some association of depressive symptoms with non-disclosure $(70 \%$ of those who had not disclosed had depressive symptoms compared to $53 \%$ among those who had disclosed). There is a high prevalence of depressive symptoms among adult patients on HAART. There is need for in-depth evaluation to find out the root causes of depressive symptoms among HAART patients in AIDSRelief clinics. There is need to integrate mental health management in HIV care and treatment as well as training the existing health workers on mental health management.

\section{Introduction}

Depression is common among persons liv- ing with HIV/AIDS in Sub-Saharan Africa (SSA). ${ }^{1}$ Prevalence of depression among people living with HIV (PLHIV) is between 21\% and $63.3 \%$ in low income settings..$^{2-5}$ In studies done in the general population in SSA depression prevalence was found to be between 9$20 \%$. In high income countries the prevalence of depression in PLHIV was found to be between $15-34 \% .{ }^{6}$ PLHIV have nearly twice the risk of developing depression and this affects their quality of life. ${ }^{7}$ Moreover, whilst depression prevalence in PLHIV is higher than in the general population, the mental health of PLHIV is generally overlooked.$^{8,9}$ Although depression is treatable, only less than $50 \%$ of patients with depression are diagnosed and treated. ${ }^{10}$ Low income countries face the challenge of channeling the few health resources towards mental health which is a low priority when compared with the burden and threat of infectious diseases. ${ }^{11,12}$ The signs and symptoms of depression are similar in HIV-infected and non-infected patients, but patients with HIV infection may more frequently have sleep and appetite disturbances. ${ }^{4}$ Depression may alter the course of HIV infection by impairing immune function or influencing behavior. ${ }^{2,4}$ It also has a negative effect on patients' sexual risk behavior and substance abuse. ${ }^{10}$ Depression is linked to poor self care practices among PLHIV and increased social isolation. ${ }^{5,8}$

In PLHIV with chronic depression, CD4 cells decrease, viral load increases and they are also prone to a higher risk of clinical decline and mortality. $2,4,8,13-15$ In a study carried out in Uganda the prevalence of depression symptoms among PLHIV being initiated on antiretroviral therapy (ART) and followed up at 3 and 6 months was almost twice (53.9\% vs $28 \%$ ) that of HIV negative controls. ${ }^{16}$

Although depression in the general population is highly prevalent in Uganda, it is rarely recognized as such. ${ }^{16}$ Depression in PLHIV has been linked to low adherence to antiretroviral therapy. ${ }^{2-5,8,12,17-22}$ This is because patients feel discouraged as a result of negative thoughts and hopelessness. ${ }^{7}$ Lack of access to resources and gender inequalities have a strong bearing on depression in PLHIV particularly women., ${ }^{2,423}$ Depression has also been linked to stigma. ${ }^{10,23}$ In other studies low spiritual well-being and low perceived social support found to be a risk factor for depression in some groups of PLHIV. ${ }^{24}$

In this paper we report the prevalence of depressive symptoms among PLHIV in AIDSRelief (AR) in order to use the evidence obtained to improve quality of care. This paper seeks to contribute to the knowledge of depressive symptoms prevalence among HIV patients on HAART using the AR experience in Uganda.
Correspondence: Constance Shumba, Uganda Program, Institute of Human Virology, University of Maryland, 124 Luthuli Avenue, Bugolobi, Kampala, Uganda.

Tel. +256774534599

E-mail: konstansezw@googlemail.com

Key words: depressive symptoms, highly active antiretroviral therapy (HAART), people living with HIV (PLHIV).

Acknowledgements: we would like to thank UMSOM-IHV Uganda, the local partner treatment facilities, UMSOM-IHV Baltimore, PEPFAR Team, CDC-Uganda.

Contributions: CS, RA, study coordination and manuscript drafting; PM, study conceiving and design, manuscript coordination and drafting; RI, statistical analysis. All authors read and approved the final manuscript.

Conflict of interests: the authors declare no potential conflict of interests.

Received for publication: 3 February 2013.

Revision received: 22 August 2013.

Accepted for publication: 11 November 2013.

This work is licensed under a Creative Commons Attribution NonCommercial 3.0 License (CC BYNC 3.0).

(C) Copyright C. Shumba et al., 2013

Licensee PAGEPress, Italy

Journal of Public Health in Africa 2013; 4:e19

doi:10.4081/jphia.2013.e19

\section{Materials and Methods}

\section{Study setting and methods}

In Uganda there are about 1.1 million PLHIV and 200,000 are on treatment. ${ }^{25}$ AR Uganda program where this study was conducted has 79,989 PLHIV in care and 36,265 on HAART in 18 rural and under-served communities in the Northern, Southern, Central, Eastern and Western regions of Uganda. The program began in March 2004, working with faith-based and not-for-profit health facilities to strengthen their programming and technical capacities and applying evidence based data to improve care, treatment and community follow up.

As part of program evaluation the program adult patients 19 years and above on antiretroviral treatment for 9-15 months were selected to participate in this study. The aim of this survey was to determine the prevalence of depressive symptoms among patients with HIV on HAART in AR clinics Uganda. 


\section{Data collection}

Data was collected using the AIDSRelief adherence survey that has been piloted, validated and used in six African Countries. The survey was designed to identify specific indicators that influence adherence to antiretroviral therapy and increase in quality of life. This six component survey utilizing 46 questions was administered to the patient. Survey administrators were community health professionals trained using a unified source codebook. Components of the AR adherence survey included; family and support, assessing missed clinic appointments and/or doses, lifestyle and risk behaviors, belief in treatment, socioeconomics, support provided by clinic or hospital, depressive symptoms and mental health, assessing HIV and ART knowledge.

A sample of 732 adults was randomly selected for a patient adherence survey to assess disease, treatment knowledge and services received between August 2008 and March 2009. The number of participants selected for each site was proportional to the total number of eligible patients. Data was collected using an adherence survey questionnaire that included a depressive symptoms score. The primary variable of interest was patients' level of depressive symptoms score, constructed using factor analysis from five survey questions relating to: sadness, need to be alone, hopelessness and confusion.

Depressive symptoms were measured from five questions related to mental health issues in the survey: i) In the past month, have you had persistent feelings of sadness or hopelessness that you just cannot control?; ii) How often do you feel the need to be alone?; iii) In the last month (or something better); have you lost interest in what used to give you pleasure?; iv) In the past month, how often have you felt confused or not yourself?; and v) How often do you feel that life is too difficult for you to go on? The responses for all these five questions were always, sometimes, never. The data coded responses of always as 2 sometimes 1 and never as 0 . A summative score was created ranging from 0 and 10.

\section{Statistical analysis}

The total scores were then further categorized through the confirmatory factor analysis method (STATA version 11) into four categories; no depressive symptoms, low depressive symptoms, medium depressive symptoms and high depressive symptoms. The reliability of this scale in the study was at a Cronbach's alpha of 0.87 .

This survey was done as part of a routine quality improvement program. Adherence counselors and nurses at the AR clinics were trained to administer the questionnaire. Informed consent form was provided to the patients and after full explanation from the health staff about the survey, the patients would read through the consent and clarity provided. The patients appended his or her signature indicating consent to participate in the survey. Each patient had the right to withdraw at anytime in the course of the survey.

\section{Results}

Of the 732 adults who participated in the survey, more than half (69\%) were women. Most of the participants were aged between 1950 years and majority had primary level education. About $90 \%$ of the participants who responded to the disclosure question had disclosed their HIV status.

Overall depressive symptoms prevalence was $59 \%$ and $26 \%$ experienced low depressive symptoms, 18\% medium depressive symptoms and $14 \%$ high depressive symptoms. Women had a higher depressive symptoms score than men (66\% vs 43\%) and this was statistically significant at univariate analysis. Non-disclosure of HIV status showed some association with depressive symptom scores. Age and education level were not associated with depressive symptoms scores (Table 1).

\section{Discussion}

The study found a high (59\%) prevalence of depressive symptoms amongst PLHIV in AR in Uganda. This is consistent with other studies which also found a prevalence of $57 \%{ }^{2,4}$ The survey was representative of the wider adult population experiences as it included both men and women unlike most studies on mental health of PLHIV which focus on subgroups thus lacking direct relevance to the wider population experiences. ${ }^{22}$ This high level of depression maybe due to the fact that most AR patients are mostly rural, have a low socio-economic status and do not have access to resources. ${ }^{2}$ Patients with many social and medical needs are more likely to have a diagnosis of depression. ${ }^{7}$ Most of the PLHIV seen in AR clinics are women (69\%) and this was consistent with another finding that women are a majority in HIV care and at the same time are more prone to depression. ${ }^{16}$ This might also explain the high prevalence of depressive symptoms found in AR clinics of $58 \%$.

Although depression is a serious debilitating illness in some parts of the world it is linked to western cultures and is believed to have low public health importance in some cultures due to the indirect link with mortality. ${ }^{11}$ Depressive symptoms in Buganda are conceptualized as a problem related to thinking too much illness of thoughts resulting from various socioeconomic problems and requires no medication for thoughts. ${ }^{17}$ This may also be true for AR patients who may not see this as a problem requiring medical intervention. Depression was found to be rarely diagnosed in HIV treatment settings in the US. ${ }^{5}$ This is also true for AR where there is no routine screening for depressive symptoms.

Although some research on depression in PLHIV attributes it to lack of access to HAART and being on treatment for a short period of time. $^{23}$ The length of time itself was not defined, however we found that even though AR patients surveyed had been on HAART for 9-15 months, they still had a high prevalence of depressive symptoms. This suggests that depressive symptoms are transient and ongoing even after being on HAART for consider-

Table 1. Factors associated with depressive symptoms among patients on highly active antiretroviral therapy.

\begin{tabular}{|c|c|c|c|c|}
\hline \multirow[b]{2}{*}{ Demographics } & \multirow{2}{*}{$\begin{array}{l}\text { Total } \\
\text { N (\%) }\end{array}$} & \multicolumn{2}{|c|}{ Depression } & \multirow[t]{2}{*}{ P-value } \\
\hline & & $\begin{array}{c}\text { Yes } \\
\text { N (\%) }\end{array}$ & $\begin{array}{c}\text { No } \\
\text { N (\%) }\end{array}$ & \\
\hline $\begin{array}{l}\text { Gender } \\
\text { Male } \\
\text { Female }\end{array}$ & $\begin{array}{l}228(31.15) \\
504(68.85)\end{array}$ & $\begin{array}{c}98(42.98) \\
331(65.67)\end{array}$ & $\begin{array}{c}130(57.02) \\
173(34.33)\end{array}$ & $<0.001$ \\
\hline $\begin{array}{l}\text { Disclosure* } \\
\text { Not disclosed } \\
\text { Disclosed }\end{array}$ & $\begin{array}{c}30(10.35) \\
260(89.65)\end{array}$ & $\begin{array}{c}21(70.00) \\
137(52.69)\end{array}$ & $\begin{array}{c}9(30.00) \\
123(47.31)\end{array}$ & 0.071 \\
\hline $\begin{array}{c}\text { Age group* } \\
19-39 \\
40-50 \\
50+\end{array}$ & $\begin{array}{c}294(43.55) \\
290(42.96) \\
91(13.48)\end{array}$ & $\begin{array}{c}183(62.24) \\
168(57.93) \\
49(53.85)\end{array}$ & $\begin{array}{c}112(37.76) \\
122(42.07) \\
42(46.15)\end{array}$ & - \\
\hline $\begin{array}{l}\text { Education level* } \\
\text { Primary } \\
\text { Secondary } \\
\text { University }\end{array}$ & $\begin{array}{c}363(53.70) \\
261(38.61) \\
52(7.69)\end{array}$ & $\begin{array}{l}218(60.06) \\
158(60.54) \\
24(46.15)\end{array}$ & $\begin{array}{l}145(39.94) \\
103(39.46) \\
28(53.85)\end{array}$ & 0.138 \\
\hline
\end{tabular}


ably long enough to start improving clinically and virologically. ${ }^{26}$ However, Nakasujja et al. who reported that depressive symptoms improved with HAART initiation. ${ }^{16}$ Their study found a decrease in depressive symptoms with increased functionality from HAART initiation up to 6 months of follow-up; $53.9 \%$ at HAART initiation, $36 \%$ at 3 months, and $30 \%$ at 6 months suggesting that access to HAART may improve depressive symptoms in the follow up phase. However, the study was very small with only 102 PLHIV study participants.

In another study carried out in Uganda there was $82.6 \%$ prevalence of psychiatric disorder among HIV patients including depression. ${ }^{27}$ However, it was not clear whether the participants were on HAART and their duration on HAART. In addition the study had a very small sample size of forty-six. In another study when compared to non-depressed patients, those with sub-clinical depression had more likely been using ART for less than one year and had advanced HIV disease. ${ }^{3}$ We did not make this comparison in our study.

In a study conducted in Uganda by Wilk and Bolton they found that some of the mental health consequences of HIV were self-hatred and self-pity. ${ }^{5}$ It is suggested that individual coping mechanisms might have influence on how PLHIV experience depressive symptoms. ${ }^{5}$ In one study it was found that patients were most likely to be virologically suppressed if they did not have a mental health diagnosis providing a strong argument for treating depressed PLHIV with HAART in view of improving outcomes. ${ }^{8}$ However, in the AR PLO process all the patients had already been on HAART for 9-15 months. This suggests that whilst HAART may be effective in reducing depressive symptoms there remains a colossal need for treating depression as a co-morbid condition. ${ }^{8}$

In areas with high HIV prevalence community led group interpersonal psychotherapy was found to reduce symptoms of depression and improve the quality of life of PLHIV. ${ }^{7,11}$ This reinforces the importance of support groups. ${ }^{5}$ There are culturally accepted ways of dealing with and healing depression and these have to be explored in every context and applied. ${ }^{17}$ Targeted intervention could slow progression to AIDS. ${ }^{13}$ There is need to identify barriers to seeking care for depressive symptoms among PLHIV. In most of the depression among PLHIV studies identified in review of literature most of the patients were not on HAART. Early recognition of risk factors and their redirection amongst PLHIV could reduce the emergence of depression. It is important to integrate mental health services in routine HIV care and encourage approaches that provide comprehensive care for PLHIV.

At the policy level there is need to develop an essential package of HIV care that includes mental health services whilst ensuring relevant and appropriate human resource capacity at the primary care level through training and task shifting. This also calls for sustained investments in mental health at the policy and implementation levels so as to ensure high quality care in scaling up HIV services using an integrated health systems approach. Building and relying on a robust evidence base is critical in developing targeted strategies for addressing mental health challenges among PLHIV. This also implies that it is necessary to engage PLHIV and their families in mental health programs.

More research needs to be done on the impact of HIV treatment on mental health. In addition, there may also be need to explore the meanings of depression in communities in Uganda. Further research must consider the qualitative and phenomenological perspectives of PLHIV and sub groups on depression as this may be useful in designing interventions. Qualitative in-depth studies need to be conducted on the characteristics of depression and the root causes in PLHIV on the AR program.

\section{Limitations}

We did not explore the causes of the depressive symptoms in greater depth so cannot attribute the depressive symptoms to any particular factors. We also did not assess depressive symptoms prior to and at HAART initiation so cannot draw conclusions on whether HAART has an impact on depressive symptoms. The survey was carried out amongst AR patients who may have some unique characteristics and may not be generalized to all patients on HAART for the same period of time in elsewhere.

\section{Conclusions}

Effective diagnosis and treatment of depression may be critical to maximizing the benefits of HIV treatment with regard to both HIV prevention and restoring the social and economic health of persons living with HIV. Linking HIV care to economic empowerment programs is necessary to reduce depression associated with lack of resources. Health workers including counselors need to be trained to recognize depressive symptoms and refer appropriately. Other mental health models of care and taskshifting could be explored.

\section{References}

1. Wagner GJ, Holloway I, Ghosh-Dastidar B, et al. Understanding the influence of depression on self-efficacy, work status and condom use among HIV clients in Uganda. J Psychosom Res 2011;70:440-8.

2. Wu DY, Munoz M, Espiritu B, et al. Depression among impoverished HIV-positive women. J Acquir Immune Defic Syndr 2008;48:500-4.

3. Nakimuli-Mpungu E, Musisi S, Katabira E, et al. Prevalence and factors associated with depressive disorders in an HIV+ rural patient population in southern Uganda. J Affect Disord 2011;135:160-7.

4. Penzak SR, Reddy YS, Grimsley SR. Depression in patients with HIV infection. Transcult Psychiatry 2006;43:287-313.

5. Collins PY, Holman AR, Freeman MC, Patel V. What is the relevance of mental health to HIV /AIDS care and treatment programs in developing countries? A systematic review. AIDS 2006;20:1571-82.

6. Ownby R, Jacobs RJ, Waldrop-Valverde D, Gould F. Depression care and prevalence in HIV positive individuals. Neurobehav HIV Med 2010:1:73-83.

7. Starace F, Ammassari A, Trotta MP, et al. Depression is a risk factor for suboptimal adherence to highly active antiretroviral therapy. J Acquir Immune Defic Syndr 2002;31:5136-9.

8. Morrison SD. Levels of self-reported depression among HIV positive patients in Albania: a cross-sectional study. Croat Med J 2011;52:622-8.

9. Hartzell JD, Janke IE, Weintrob AC. Impact of depression on HIV outcomes in the HAART era. J Antimicrob Chemother 2008; 62:246-55.

10. Patel V, Araya R, Bolton P. Treating depression in the developing world. Trop Med Int Health 2004;9:539-41.

11. Baingana FK, Alem A, Jenkins R. Mental health and the abuse of alcohol and controlled substances in disease and mortality in Sub-Saharan Africa. In: Jamison DT, Feachem RG, Makgoba MW, eds. Disease and mortality in Sub-Saharan Africa. 2nd ed. Washington, DC:World Bank Publ.; 2006. Chapter 22.

12. Leserman J. Role of depression, stress and trauma in HIV disease progression. Psychosom Med 2008;70:539-45.

13. Antelman G, Kaaya S, Wei R, et al. Depressive symptoms increase risk of HIV disease progression and mortality among women in Tanzania. $\mathrm{J}$ Acquir Immune Defic Syndr 2007;44:470-7.

14. Cook J. Depressive symptoms increase AIDS-related death. Am J Public Health 2004;150:85-94.

15. Paterson DL, Swindells S, Mohr J, et al. Adherence to protease inhibitor therapy and outcomes in patients with HIV infection. Ann Intern Med 2000;133:21-30.

16. Nakasujja N, Skolasky RL, Musisi S, et al. 
Depression symptoms and cognitive function among individuals with advanced HIV infection initiating HAART in Uganda. BMC Psychiatry 2010;10:44.

17. Okello ES, Ekblad S. Lay concepts of depression among the Baganda of Uganda: a pilot study. J Affect Disord 2011;135:160-7.

18. Ramadhani HO, Thielman NM, Landman $\mathrm{KZ}$, et al. Predictors of incomplete adherence, virologic failure and antiviral resistance among HIV infected adults receiving therapy in Tanzania. Clin Infect Dis 2007;45:1492-8.

19. Gordillo V, del Amo J, Soriano V, GonzálezLahoz J. Sociodemographic and psychological variables influencing adherence to antiretroviral therapy. AIDS 1999;13:1763-9.

20. Byakika-Tusiime J, Crane J, Oyugi JH, et al. Longitudinal antiretroviral adherence in HIV+ve Ugandan parents and their children initiating HAART in the MTCT-Plus Family Treatment Model: role of depression in declining adherence over time. AIDS Behav 2009;13 Suppl 1:82-91.

21. Rabkin JG. HIV and depression: 2008 review and update. Curr HIV/AIDS Rep 2008;5:163-71.

22. Phyllips J, Gettes D, Douglas S, et al. Sleep disturbance and depression as barriers to adherence. Am J Psychiatry 2002;14:27380.

23. Brandt R. The mental health of people living with HIV/AIDS in Africa: a systematic review. Afr J AIDS Res 2009;8:123-33.

24. Yi MS, Mrus JM, Wade TJ, et al. Religion, spirituality and depressive symptoms in patients with HIV/AIDS. J Gen Intern Med 2006;21:521-7.

25. UNAIDS. UNGASS Guidelines on construction of core indicators for 2010 reporting. Geneva: UNAIDS; 2009. Available from: http://www.unaids.org/en/resources/presscentre/featurestories/2009/march/2009033 lungass2010/

26. Smith CJ, Staszewski S, Sabin C, et al. Use of viral load measured after 4 weeks of highly active antiretroviral therapy to predict virologic outcome at 24 weeks for HIV1-positive individuals. J Acquir Immune Defic Syndr 2004;37:1155-9.

27. Petrushkin H, Boardman J, Ovuga E. Psychiatric disorders in HIV positive individuals in urban Uganda. Psychiatrist 2005;29:455-8. 